\title{
Numerical simulation of nonlinear chromatography with core-shell particles applying the general rate model
}

\author{
Shamsul Qamar, ${ }^{\mathrm{a}, \mathrm{b}, *}$, Fouzia Abdul Sattar ${ }^{\mathrm{b}}$, Javeria Nawaz Abbasi ${ }^{\mathrm{b}}$, Andreas \\ Seidel-Morgenstern ${ }^{\mathrm{a}}$ \\ ${ }^{a}$ Max Planck Institute for Dynamics of Complex Technical Systems Magdeburg, Germany \\ ${ }^{b}$ Department of Mathematics, COMSATS Institute of Information Technology, Islamabad, Pakistan
}

\begin{abstract}
Core-shell particles allow highly efficient and fast separation of complex samples. They provide advantages over fully porous particles, such as highly efficient separation with fast flow rate due to shorter diffusional path length in particle macropores. On the other hand, capacities are reduced due to the inert core. This work is focused on the numerical approximation of a nonlinear general rate model for fixed-beds packed with core-shell particles. The model equations consider axial dispersion, interfacial mass transfer, intraparticle diffusion, and multi-component Langmuir isotherm. A semi-discrete high resolution fluxlimiting finite volume scheme is proposed to accurately and efficiently solve the model equations. The scheme is second order accurate in axial and radial coordinates. The resulting system of ordinary differential equations (ODEs) are solved by using a second-order TVD Runge-Kutta method. For illustration, a few selected scenarios of single solute and multi-component elution bands are generated to study theoretically the effects of the core radius fractions on the course of elution curves. Typically applied performance criteria are
\end{abstract}

\footnotetext{
${ }^{*}$ Corresponding author. Tel: +92-51-90495413; fax: +92-51-4442805

Email addresses: shamsul.qamar@comsats.edu.pk (Shamsul Qamar)
} 
evaluated for identifying ranges of optimum values of the core radius fraction.

Key words: Dynamics of chromatography, nonlinear general rate model, core-shell particles, adsorption, mass transfer.

\section{Introduction}

High performance liquid chromatography (HPLC) is considered to be an essential analysis tool for research, manufacturing, clinical tests, and diagnostics. The current trends in HPLC are towards achievement of higher kinetic efficiency, shorter analysis time and low back pressure for various types of samples. In efforts to increase the power of HPLC, the particles packed into the columns have been progressively improved and downsized. The development of ultra-high-performance liquid chromatography (UHPLC) using such smaller particles has made possible to enhance the good analytical features of HPLC and to improve the column performance. However, despite the fact that UHPLC allows efficient and fast separations and utilizes smaller amounts of solvents, the small sized particles put an extra burden on HPLC instrumentation, see Salisbury (2008). The invention of core beads (also known as pellicular, superficially porous, and fused-cored beads or particles) has introduced an alternative technology which avoids the use of special equipments. Their efficiency is comparable to UHPLC and have low back pressures, see Kirkland et al. (2000); Fanigliulo et al. (2010); Pietrogrande et al. (2010). They also provide the possibility of using already existing conventional HPLC systems in the laboratories, see Ali et al. (2012). Cored beads are made of a solid core surrounded by a porous layer having essentially the properties of porous particles used in conventional HPLC columns, see Horvath et al. 
(1967); Pesek et al. (1973); Ning et al. (1998); Coutinho et al. (2001); Greibrokk (2004); Kirkland et al. (2000, 2007); Zhou et al. (2007); Kiss (2010); Manchon et al. (2010); Gritti et al. (2010a,b); Guiochon and Gritti (2011); Spudeit et al. (2014); Hayes et al. (2014); Lambert et al. (2014); González-Ruiz et al. (2015); Horváth and Felinger (2015). They were introduced to build highly efficient HPLC columns for separating the constituents of high molecular weight compounds of biological origin, see e.g. Horvath et al. (1967). They offer advantages over fully porous beads, such as highly efficient separation with fast flow rate due to shorter diffusional path length in particle macropores. For compounds having high internal mass-transfer resistance, the gain in efficiency compensates the reduction in sample capacity due to the lower volume of porous adsorbent, see Kaczmarski and Guiochon (2007). On the other hand, the gain decreases with decreasing mass-transfer resistance, for instance with decreasing molecular weight of the compound.

In HPLC, the use of cored beads is a compromise between fully porous beads and nonporous beads, see Wang et al. (2006). Non-porous beads eliminate intraparticle diffusion and generate sharp elution peaks of the shortest retention times, see Lee (1997); Rissler (2000); Xiang et al. (2003); Fekete et al. (2010); Gu et al. (2011). However, they are unable to provide sufficient retention time range in HPLC due to their limited binding capacities, see e.g. Kirkland et al. (2000); Miyabe (2008). In contrast, fully porous beads offer the largest retention time differences, but suffering from excessive band broadening. Fanigliulo et al. (2010) and Cabootera et al. (2010) performed experiments to compare and analyze several commercially available fully porous and cored beads.

Mathematical modeling of the dynamic chromatographic process is an important ingredient 
of the chromatography theory. It offers a technique for predicting the dynamic behaviors of the solute in the columns without extensive experiments. Because of different levels of complexity, several models have been established and applied in the literature, see Ruthven (1984); Carta (1988); Guiochon (2003); Guiochon and Lin (2003). Among them, the General Rate model (GRM) is the most complicated and complete. It includes most of the factors which influence mass transfer process in the column, such as the axial dispersion, external mass transfer resistance, pore diffusion, surface diffusion and adsorption/desorption kinetic procedure. Thus, GRM possesses the potential to include more kinetic resistances, which makes it more realistic. For certain limits of transport parameters it converges into the LKM, see Guiochon et al. (2006). Zhou et al. (2007) used a general rate model for cored beads to obtain intraparticle diffusion coefficients. Kaczmarski and Guiochon (2007) considered thin-shelled coated beads using a lumped particle model. They assumed that the concentration profile inside the thin shell could be expressed by a single averaged concentration value. For comparison with fully porous beads, they used the general rate model for fully porous beads. Li et al. (2010) performed optimization of core size for linear chromatography by minimizing height equivalent theoretical plate (HETP) number. Gu et al. (2011) have presented a nonlinear general rate model for cored beads and its numerical solution strategy. The model was used to optimize the core radius fraction for a preparative ternary elution system as an example case. Luo et al. (2013) have simulated cored beads for size-exclusion chromatography using the general rate model (GRM). Li et al. (2003a) have used GRM and Langmuir isotherm to analyze adsorption/desorption kinetics of protein on the binding ligand of inert core adsorbent. Moreover, Li et al. (2003b, 2004) 
have derived analytical solution of the linear GRM to predict breakthrough curves for the inert core adsorbent. Core-shell particles find one real application at industrial scale on expanded bed adsorption for protein capture. The use of similar model and application has been discussed by Li et al. (2005, 2006).

This article is concerned with the numerical approximation of a nonlinear GRM for coreshell particles. It is an extension of our recent work on the analysis of linear GRM for coreshell particles, see Qamar et al. (2015). For nonlinear adsorption isotherms, analytical solutions of the model equations are not possible. For that reason, numerical simulations are required to accurately predict the dynamic behavior of chromatographic columns. Steep concentration fronts may occur because of the convection dominated partial differential equations (PDEs) of the chromatographic models. Thus, efficient and accurate numerical method is required to study dynamics in chromatographic columns packed with core-shell particles. The model considers multi-component mixture, axial dispersion, interfacial mass transfer, intraparticle diffusion, and nonlinear adsorption. A high resolution flux-limiting finite volume scheme is suggested to solve the model equations. The accuracy and efficiency of this scheme for equilibrium dispersive model was recently tested by Javeed et al. (2011). In that article, it was found that the proposed scheme is simple, accurate, and easy to implement as compared to other high resolution finite volume and finite element methods applied to the same model equations. Different case studies of the one-, two-, and three-component mixtures are considered. With these case studies it is intended to analyze the effects of core radius fraction, axial dispersion, film mass transfer resistance and intraparticle diffusion resistance, on the elution curves. Assessment criteria are introduced to 
understand the process and to optimize the core size for achieving maximum productivity. A comparison of linear and nonlinear cases is presented to reveal the effects of nonlinearity. The tools developed and the simulation results generated should be helpful to synthesize tailor-made particles.

This article is arranged as follows. In Section 2, the nonlinear general rate model using cored beads is briefly introduced. In Section 3, the proposed accurate and efficient numerical scheme is derived and criteria to evaluate the performance of preparative chromatography are introduced. In Section 4, several case studies are carried out which are helpful in rationally designing thicknesses of shell layers for preparative applications. Finally, conclusions are drown in Section 5

\section{Mathematical model for core beads}

An isothermal adsorption column packed with inert core particles is considered as shown in Figure 1. At time zero, a step change in the concentration of an adsorbate is introduced in a flowing stream. The adsorption column is subjected to axial dispersion, film mass transfer resistance and intraparticle diffusion resistance. It is assumed that cored beads

have uniform particle size $R_{p}$ and core size $R_{\text {core }}$. The inner core cannot be penetrated and there is only diffusion (no convection) in the porous shell. The column is considered to be isothermal.

Under these assumptions, the mass balance equations for a multi-component mixture of $N_{c}$ components percolating through a column filled with spherical core beads of radius $R_{p}$ 
are given as (c.f. Guiochon (2003); Guiochon et al. (2006))

$$
\frac{\partial C_{b, i}}{\partial t}+u \frac{\partial C_{b, i}}{\partial z}=\quad D_{b, i} \frac{\partial^{2} C_{b, i}}{\partial z^{2}}-\frac{3}{R_{p}} F_{b} k_{\text {ext }}\left(C_{b, i}-\left.C_{p, i}\right|_{r=R_{p}}\right), \quad i=1,2, \cdots, N_{c} .
$$

In the above equation, $C_{b, i}$ and $C_{p, i}$ are the concentrations of $i$-th component of the mixture in the bulk of the fluid and in particle pores, respectively. The phase ratio $F_{b}$ is defined as $F_{b}=\left(1-\epsilon_{b}\right) / \epsilon_{b}$, where $\epsilon_{b}$ is the external porosity. Moreover, $u$ is the interstitial velocity which respects the external porosity, $D_{b, i}$ represents the axial dispersion coefficient of the $i$-th component, $k_{\text {ext }}$ is the external mass transfer coefficient, and $t$ and $z$ denote time and axial coordinate of the column. In addition, $r$ denotes the radial coordinate (c.f. Figure 1). The mass balance equation for the solute in the stationary phase can be expressed as (c.f. Guiochon (2003); Guiochon et al. (2006))

$$
\epsilon_{p} \frac{\partial C_{p, i}}{\partial t}+\left(1-\epsilon_{p}\right) \frac{\partial q_{p, i}}{\partial t}=\frac{\epsilon_{p} D_{p, i}}{r^{2}} \frac{\partial}{\partial r}\left(r^{2} \frac{\partial C_{p, i}}{\partial r}\right)
$$

where $q_{p, i}$ is the local concentration of $i$-th component of the mixture in the shell of the stationary phase, $\epsilon_{p}$ is the internal porosity, and $D_{p, i}$ is the pore diffusivity of $i$-th component of the mixture.

To simplify the notations and reduce the number of variables, the following dimensionless quantities are introduced:

$$
\begin{aligned}
& c_{b, i}=\frac{C_{b, i}}{C_{b, i}^{\mathrm{inj}}}, \quad c_{p, i}=\frac{C_{p, i}}{C_{b, i}^{\mathrm{inj}}}, \quad q_{p, i}^{*}=\frac{q_{p, i}}{C_{b, i}^{\mathrm{inj}}}, \quad \tau=\frac{u t}{L}, \quad \rho=\frac{r}{R_{p}}, \quad x=\frac{z}{L}, \\
& P e_{i}=\frac{L u}{D_{b, i}}, \quad B i_{i}=\frac{k_{\mathrm{ext}} R_{p}}{\epsilon_{p} D_{p, i}}, \quad \eta_{i}=\frac{\epsilon_{p} D_{p, i} L}{R_{p}^{2} u}, \quad \xi_{i}=3 B i_{i} \eta_{i} F_{b} .
\end{aligned}
$$

In the above equation, $C_{b, i}^{\text {inj }}$ denotes the non-zero injected bulk concentration of $i$-th component, $P e_{i}$ is the Peclet number based on column length, $B i_{i}$ represents the modified Biot 
number, and $\eta_{i}$ describes the ratio of space time and interaparticle diffusion time for the $i$-th component. Using the above dimensionless variables, the model Eqs. (1) and (2) can be rewritten as

$$
\begin{gathered}
\frac{\partial c_{b, i}}{\partial \tau}+\frac{\partial c_{b, i}}{\partial x}=\frac{1}{P e} \frac{\partial^{2} c_{b, i}}{\partial x^{2}}-\xi_{i}\left(c_{b, i}-\left.c_{p, i}\right|_{\rho=1}\right) \\
\epsilon_{p} \frac{\partial c_{p, i}}{\partial t}+\left(1-\epsilon_{p}\right) \frac{\partial q_{p, i}^{*}}{\partial t}=\frac{\eta_{i}}{\rho^{2}} \frac{\partial}{\partial \rho}\left(\rho^{2} \frac{\partial c_{p, i}}{\partial \rho}\right) .
\end{gathered}
$$

Eqs. (4) and (5) are connected at $r=R_{p}$ via the following expression which quantifies the temporal change of the average loading of the particles:

$$
\left.\frac{\partial c_{p, i}}{\partial \rho}\right|_{\rho=1}=B i_{i}\left(c_{b, i}-\left.c_{p, i}\right|_{\rho=1}\right) .
$$

The corresponding convex nonlinear Langmuir isotherm in dimensionless form is given as

$$
q_{p, i}^{*}=\frac{a_{i} c_{p, i}}{1+\sum_{j=1}^{N_{c}}\left(b_{j} C_{b, j}^{\mathrm{inj}}\right) c_{p, j}} .
$$

For fully porous particles $\rho$ ranges from 0 to 1 , while for cored particles it ranges from $\rho_{\text {core }}=R_{\text {core }} / R_{p}$ to 1 . As this study is concerned with the cored particles of arbitrary core radius fraction $\rho_{\text {core }}$, it is necessary to allow the core radius to be changed. For cored particles (c.f. Eq. (5)), $\rho_{\text {core }} \leq \rho \leq 1$. For fully porous particles $\rho_{\text {core }}=0$, while $\rho_{\text {core }} \neq 0$ for cored particles. Thus, following $\mathrm{Gu}$ et al. (2011), it is helpful to replace $\rho$-axis by $0 \leq \gamma \leq 1$, where

$$
\gamma=\frac{\rho-\rho_{\text {core }}}{1-\rho_{\text {core }}}
$$


On substituting

$$
\rho=\gamma\left(1-\rho_{\text {core }}\right)+\rho_{\text {core }}
$$

in Eqs. (4) and (5) and using Eq. (7), they yield

$$
\begin{aligned}
& \frac{\partial c_{b, i}}{\partial \tau}+\frac{\partial c_{b, i}}{\partial x}=\frac{1}{P e_{i}} \frac{\partial^{2} c_{b, i}}{\partial x^{2}}-\xi_{i}\left(c_{b, i}-\left.c_{p, i}\right|_{\gamma=1}\right), \quad i=1,2, \cdots, N_{c} \\
& \left(\epsilon_{p}+\left(1-\epsilon_{p}\right) \frac{\partial q_{p, i}^{*}}{\partial c_{p, i}}\right) \frac{\partial c_{p, i}}{\partial \tau}=\eta_{b}\left(\frac{1}{\left(1-\rho_{\text {core }}\right)^{2}} \frac{\partial^{2} c_{p, i}}{\partial \gamma^{2}}+\frac{2}{\gamma\left(1-\rho_{\text {core }}\right)^{2}+\rho_{\text {core }}\left(1-\rho_{\text {core }}\right)} \frac{\partial c_{p, i}}{\partial \gamma}\right)
\end{aligned}
$$

The Eqs. (10) and (11) are also subjected to the initial and boundary conditions. The initial conditions for an initially regenerated column are given as

$$
c_{b, i}(0, x)=0, \quad c_{p, i}(0, x, \gamma)=0, \quad \forall x, \gamma \in(0,1), \quad i=1,2, \cdots, N_{c} .
$$

The following boundary conditions at $\gamma=0$ and $\gamma=1$ are assumed for Eq. (11) (c.f. Eq. (6))

$$
\left.\frac{\partial c_{p, i}}{\partial \gamma}\right|_{\gamma=0}=0,\left.\quad \frac{\partial c_{p, i}}{\partial \gamma}\right|_{\gamma=1}=\left(1-\rho_{\text {core }}\right) B i_{i}\left(c_{b, i}-\left.c_{p, i}\right|_{\gamma=1}\right) .
$$

Appropriate inlet and outlet boundary conditions (BCs) are required for Eq. (10). In this case, the Robin type boundary condition, known in chemical engineering as Danckwerts boundary conditions, are applied at the column inlet (c.f. Danckwerts (1953))

$$
-\frac{1}{P e_{i}} \frac{\partial c_{b, i}}{\partial x}+\left.c_{b, i}\right|_{x=0}=\left\{\begin{array}{rrr}
1, & \text { if } 0<\tau \leq \tau_{\text {inj }} \\
0, & \tau>\tau_{i n j}
\end{array}\right.
$$

where $\tau_{\text {inj }}$ is the time of injection. At the outlet of the column of finite length $x=1$, the following Neumann outflow boundary conditions are used:

$$
\frac{\partial c_{b, i}(1, \tau)}{\partial x}=0
$$




\section{Numerical scheme}

Various numerical schemes are available in the literature to approximate the chromatographic models, see Javeed et al. (2011); Guiochon and Lin (2003); Guiochon et al. (2006); Lieres (2010) and reference therein. In this work, a semi-discrete high resolution fluxlimiting finite volume scheme is applied to solve the current model equations in axial- and radial-coordinates. This scheme was tested recently for equilibrium dispersive model by Javeed et al. (2011). In that article, it has been shown analytically and numerically that the proposed scheme is second order accurate, see Javeed et al. (2011). A second-order TVD Runge-Kutta method is applied to solve the ODE system in time-coordinate.

By considering the Langmuir isotherm in Eq. (7) and a three-component mixture (i.e. $N c=3$ ), we deduce the following system of equations from dimensionless Eqs. (10) and

$$
\begin{aligned}
\frac{\partial \mathbf{c}_{b}}{\partial \tau}+\frac{\partial \mathbf{c}_{b}}{\partial x} & =\mathbf{P} \frac{\partial^{2} \mathbf{c}_{b}}{\partial x^{2}}-\xi\left(\mathbf{c}_{b}-\left.\mathbf{c}_{p}\right|_{r=1}\right), \\
\mathbf{J} \frac{\partial \mathbf{c}_{p}}{\partial \tau} & =\eta\left(\frac{1}{\left(1-\rho_{\text {core }}\right)^{2}} \frac{\partial^{2} \mathbf{c}_{p}}{\partial \gamma^{2}}+\frac{2}{\gamma\left(1-\rho_{\text {core }}\right)^{2}+\rho_{\text {core }}\left(1-\rho_{\text {core }}\right)} \frac{\partial \mathbf{c}_{p}}{\partial \gamma}\right),
\end{aligned}
$$

where

$$
\begin{aligned}
& \mathbf{c}_{b}=\left[\begin{array}{c}
c_{b, 1} \\
c_{b, 2} \\
c_{b, 3}
\end{array}\right], \mathbf{c}_{p}=\left[\begin{array}{c}
c_{p, 1} \\
c_{p, 2} \\
c_{p, 3}
\end{array}\right], \mathbf{P}=\left[\begin{array}{ccc}
\frac{1}{P e_{1}} & 0 & 0 \\
0 & \frac{1}{P e_{2}} & 0 \\
0 & 0 & \frac{1}{P e_{3}}
\end{array}\right], \xi=\left[\begin{array}{ccc}
\xi_{1} & 0 & 0 \\
0 & \xi_{2} & 0 \\
0 & 0 & \xi_{3}
\end{array}\right], \\
& \mathbf{J}=\left[\begin{array}{ccc}
1+F_{b} \frac{\partial q_{p, 1}}{\partial c_{p, 1}} & F_{b} \frac{\partial q_{p, 1}}{\partial c_{p, 2}} & F_{b} \frac{\partial q_{p, 1}}{\partial c_{p, 3}} \\
F_{b} \frac{\partial q_{p, 2}}{\partial c_{p, 1}} & 1+F_{b} \frac{\partial q_{p, 2}}{\partial c_{p, 2}} & F_{b} \frac{\partial q_{p, 2}}{\partial c_{p, 3}} \\
F_{b} \frac{\partial q_{p, 3}}{\partial c_{p, 1}} & F_{b} \frac{\partial q_{p, 3}}{\partial c_{p, 2}} & 1+F_{b} \frac{\partial q_{p, 3}}{\partial c_{p, 3}}
\end{array}\right], \eta=\left[\begin{array}{ccc}
\eta_{1} & 0 & 0 \\
0 & \eta_{2} & 0 \\
0 & 0 & \eta_{3}
\end{array}\right] .
\end{aligned}
$$


Here, $F_{b}=\left(1-\epsilon_{b}\right) / \epsilon_{b}$, where $\epsilon_{b}$ is the external porosity. Before deriving the scheme, the first step is to discretize the computational domain.

\subsection{Domain discretization}

Let $N_{x}$ and $N_{\gamma}$ be the large integers in $x$ and $\gamma$-coordinates, respectively. We consider a domain $[0,1] \times[0,1]$ which is covered by cells $\Omega_{k l} \equiv\left[x_{k-\frac{1}{2}}, x_{k+\frac{1}{2}}\right] \times\left[\gamma_{k-\frac{1}{2}}, \gamma_{l+\frac{1}{2}}\right]$ for $1 \leq k \leq N_{x}$ and $1 \leq l \leq N_{\gamma}$. The representative coordinates in the cell $\Omega_{k l}$ are denoted by $\left(x_{k}, \gamma_{l}\right)$. Here

$$
\left(x_{1 / 2}, x_{1 / 2}\right)=(0,0), \quad x_{k}=\frac{x_{k-1 / 2}+x_{k+1 / 2}}{2}, \quad \gamma_{l}=\frac{\gamma_{l-1 / 2}+\gamma_{l+1 / 2}}{2}
$$

and for uniform mesh

$$
\Delta x=x_{k+1 / 2}-x_{k-1 / 2}, \quad \Delta \gamma=\gamma_{l+1 / 2}-\gamma_{l-1 / 2}
$$

Note that

$$
\mathbf{c}_{b}=\mathbf{c}_{b}(t, x), \quad \text { and } \quad \mathbf{c}_{p}=\mathbf{c}_{p}(t, x, \gamma)
$$

Thus, for $I_{k}=\left[x_{k-\frac{1}{2}}, x_{k+\frac{1}{2}}\right]$ and $\Omega_{k l}$, the cell averaged values $\mathbf{c}_{b, k}(t)$ and $\mathbf{c}_{p, k, l}(t)$ at any time $t$ are given as

$$
\mathbf{c}_{l}=\mathbf{c}_{l}(t)=\frac{1}{\Delta x_{k}} \int_{I_{k}} \mathbf{c}(t, x) d x, \quad \mathbf{c}_{p, l}=\mathbf{c}_{p, k, l}(t)=\frac{1}{\Delta x_{k} \Delta \gamma_{l}} \int_{\Omega_{k l}} \mathbf{c}_{p}(t, x, \gamma) d \gamma d x
$$

On integrating Eq. (15) over the interval $I_{k}$ and using Eq. (21), we obtain

$$
\frac{d \mathbf{c}_{b, k}}{d \tau}=-\frac{\left(\mathbf{c}_{b, k+1 / 2}-\mathbf{c}_{b, k-1 / 2}\right)}{\Delta x}+\frac{\mathbf{P}}{\Delta x}\left[\left(\frac{\partial \mathbf{c}_{b}}{\partial x}\right)_{k+1 / 2}-\left(\frac{\partial \mathbf{c}_{b}}{\partial x}\right)_{k-1 / 2}\right]-\xi\left(\mathbf{c}_{b, k}-\mathbf{c}_{p, k, N_{\gamma}}\right),
$$


where $k=1,2, \cdots, N_{x}$. The differential terms of the diffusion part can be approximated as

$$
\left(\frac{\partial \mathbf{c}_{b}}{\partial x}\right)_{k \pm 1 / 2}= \pm \frac{\left(\mathbf{c}_{b, k \pm 1}-\mathbf{c}_{b, k}\right)}{\Delta x}
$$

Now, integration of equation (16) over the interval $\Omega_{i j}$ gives

$$
\frac{d \mathbf{c}_{p, k, l}}{d \tau}=\frac{\mathbf{J}^{-1} \eta}{\left(1-\rho_{\text {core }}\right)^{2} \Delta \gamma}\left[\left(\frac{\partial \mathbf{c}_{b}}{\partial \gamma}\right)_{k, l+1 / 2}-\left(\frac{\partial \mathbf{c}_{b}}{\partial \gamma}\right)_{k, l-1 / 2}+\frac{2\left(\mathbf{c}_{p, k, l+1 / 2}-\mathbf{c}_{p, k, l-1 / 2}\right)}{\gamma_{l+1 / 2}+\rho_{\text {core }} /\left(1-\rho_{\text {core }}\right)}\right]
$$

where

$$
\left(\frac{\partial \mathbf{c}_{p}}{\partial x}\right)_{k, l \pm 1 / 2}= \pm \frac{\left(\mathbf{c}_{p, k, l \pm 1}-\mathbf{c}_{p, k, l}\right)}{\Delta \gamma}
$$

The next step is to approximate concentrations at the cells interfaces $x_{k \pm 1 / 2}$ and $\gamma_{l \pm 1 / 2}$ in Eqs. (22) and (24). There are several ways to approximate these fluxes, leading to different numerical schemes. Here, we present the first and second order approximations. Since all components of vectors $\mathbf{P}$ and $\frac{2 \mathbf{J}^{-1} \eta}{\Delta \gamma\left[\gamma_{l+1 / 2}\left(1-\rho_{\text {core }}\right)^{2}+\rho_{\text {core }}\left(1-\rho_{\text {core }}\right)\right]}$ are positive, the vectors of contractions $\mathbf{c}_{b}$ and $\mathbf{c}_{p}$ at the cell interfaces are approximated as follows.

\subsection{First order scheme}

In this scheme backward difference formula is used to approximate concentrations at the cell interfaces:

$$
\mathbf{c}_{b, k+\frac{1}{2}}=\mathbf{c}_{b, k}, \quad \mathbf{c}_{b, k-\frac{1}{2}}=\mathbf{c}_{b, k-1}, \quad \mathbf{c}_{p, k, l+\frac{1}{2}}=\mathbf{c}_{p, k, l}, \quad \mathbf{c}_{p, k, l-\frac{1}{2}}=\mathbf{c}_{p, k, l-1}
$$

The above approximations give first order accuracy of the scheme in the axial- and particle radial-coordinates. 


\subsection{The flux-limiting high resolution scheme}

According to this scheme the cell interface concentrations are approximated as (c.f. Javeed et al. (2011))

$$
\begin{gathered}
\mathbf{c}_{b, k+\frac{1}{2}}=\mathbf{c}_{b, k}+\frac{1}{2} \varphi\left(\mu_{k+\frac{1}{2}}\right)\left(\mathbf{c}_{b, k}-\mathbf{c}_{b, k-1}\right), \quad \mu_{k+\frac{1}{2}}=\frac{\mathbf{c}_{b, k+1}-\mathbf{c}_{b, k}+\zeta}{\mathbf{c}_{b, k}-\mathbf{c}_{b, k-1}+\zeta} \\
\mathbf{c}_{p, k, l+\frac{1}{2}}=\mathbf{c}_{p, k, l}+\frac{1}{2} \psi\left(\nu_{k, l+\frac{1}{2}}\right)\left(\mathbf{c}_{p, k, l}-\mathbf{c}_{p, k, l-1}\right), \quad \nu_{k+\frac{1}{2}}=\frac{\mathbf{c}_{p, k, l+1}-\mathbf{c}_{p, k, l}+\zeta}{\mathbf{c}_{p, k, l}-\mathbf{c}_{p, k, l-1}+\zeta}
\end{gathered}
$$

Here, $\zeta=10^{-10}$ is use to prevent division by zero. The flux limiting functions $\varphi$ and $\psi$ are used to preserve the local monotonicity (positivity) of the numerical scheme (c.f. Javeed et al. (2011)). They are given as

$$
\begin{gathered}
\varphi\left(\mu_{k+\frac{1}{2}}\right)=\max \left(0, \min \left(2 \mu_{k+\frac{1}{2}}, \min \left(\frac{1}{3}+\frac{2}{3} \mu_{k+\frac{1}{2}}, 2\right)\right)\right), \\
\psi\left(\nu_{k, l+\frac{1}{2}}\right)=\max \left(0, \min \left(2 \nu_{k, l+\frac{1}{2}}, \min \left(\frac{1}{3}+\frac{2}{3} \nu_{k, l+\frac{1}{2}}, 2\right)\right)\right) .
\end{gathered}
$$

The proposed high resolution scheme in Eqs. (27)-(30) is not applicable up to the boundary intervals. Therefore, the first order (backwards) approximations are used in the boundary intervals. The fluxes at all other interior interval are computed by using Eqs. (27)-(30). Note that, this first order scheme will not diminish the global accuracy of the method. To obtain the second order accuracy in time, we use a second order TVD Runge-Kutta scheme to solve Eqs. (27)-(30), see Javeed et al. (2011). Denoting the right-hand side of Eqs. (27) and (28) as $\mathcal{L}\left(\mathbf{c}_{b},\left.\mathbf{c}_{p}\right|_{\gamma=1}\right)$ and $\mathcal{M}\left(\mathbf{c}_{p}\right)$, a second order TVD Runge-Kutta scheme update $\mathbf{c}_{b}$ and $\mathbf{c}_{p}$ through the following two stages

$$
\begin{aligned}
\mathbf{c}_{b}^{(1)} & =\mathbf{c}_{b}^{n}+\Delta \tau \mathcal{L}\left(\mathbf{c}_{b}^{n},\left.\mathbf{c}_{p}^{n}\right|_{\gamma=1}\right), \quad \mathbf{c}_{p}^{(1)}=\mathbf{c}_{p}^{n}+\Delta \tau \mathcal{M}\left(\mathbf{c}_{p}^{n}\right) \\
\mathbf{c}_{b}^{n+1} & =\frac{1}{2}\left[\mathbf{c}_{b}^{n}+\mathbf{c}_{b}^{(1)}+\Delta \tau \mathcal{L}\left(\mathbf{c}_{b}^{(1)},\left.\mathbf{c}_{p}^{(1)}\right|_{\gamma=1}\right)\right], \quad \mathbf{c}_{p}^{n+1}=\frac{1}{2}\left[\mathbf{c}_{p}^{n}+\mathbf{c}_{p}^{(1)}+\Delta \tau \mathcal{M}\left(\mathbf{c}_{p}^{(1)}\right)\right],
\end{aligned}
$$


where $\mathbf{c}_{b}^{n}$ and $\mathbf{c}_{p}^{n}$ are solutions at the previous time step $\tau^{n}$ and $\mathbf{c}_{b}^{n+1}$ and $\mathbf{c}_{p}^{n+1}$ are updated solutions at the next time step $\tau^{n+1}$. Moreover, $\Delta \tau$ represents the time step which is calculated under the following Courant-Friedrichs-Lewy (CFL) condition

$$
\Delta \tau \leq 0.5 \min \left(\Delta x, \frac{\Delta x^{2}}{\max \left(P e_{b, k}\right)}, \frac{\Delta \gamma^{2}}{\sigma\left(\mathbf{J}^{-1} \eta_{p, k}\right)}, \frac{\rho_{\text {core }}\left(1-\rho_{\text {core }}\right) \Delta \gamma}{2 \sigma\left(\mathbf{J}^{-1} \eta_{p, k}\right)}\right)
$$

where $\sigma$ represents the spectral radius of a matrix. The suggested numerical scheme is second order accurate in time and spatial coordinates, see Javeed et al. (2011). The algorithm was implemented in the computer using $\mathrm{C}$ programming language.

\subsection{Process performance criteria}

Like the other industrial processes, preparative chromatography needs to be optimized. Here, we introduce a performance criteria which could be used for the assessment of quality of a product, see Horváth and Felinger (2015). Consider a two component mixture in which component 1 has lower affinity for the stationary phase as compared to the component 2, i.e. $a_{1}<a_{2}$. The concentration of $i$-th component in grams per liter is given as $C_{b, i}=c_{b, i} C_{b, i}^{\text {inj }}$, where $c_{b, i}$ is the dimensionless concentration normalized with $C_{b, i}^{\mathrm{inj}}$ as defined in Eq. (3). Let $\tau_{1}$ be the dimensionless time at which the fraction of component 1 exceeds some threshold, i.e. $C_{b, 1} \geq \epsilon C_{b, 1}^{\text {inj }}$, where $\epsilon=10^{-5}$ in this case. Similarly, $\tau_{2}$ is the time at which

the fraction of component 2 drops below some threshold, $C_{b, 2} \leq \epsilon C_{b, 1}^{\text {inj }}$. The cycle time $\tau_{\text {cyc }}$ is defined as the time lapse between two successive injections:

$$
\tau_{\text {cyc }}=\tau_{2}-\tau_{1}
$$


The cut time of component 1 is the time at which fractionation of the this component stops. The following equation was solved to obtain the cut time $\tau_{\text {cut }}$ of component 1 :

$$
\operatorname{Pur}=\frac{\int_{\tau_{1}}^{\tau_{\text {cut }}} C_{b, 1}(\tau, x=1) d \tau}{\int_{\tau_{1}}^{\tau_{\text {cut }}}\left[C_{b, 1}(\tau, x=1)+C_{b, 2}(\tau, x=1)\right] d \tau} .
$$

The required peak area based purity was set to $99 \%$. A reduced productivity $\operatorname{Pr}$ is the amount of desired compound produced per time cycle. For the case of component 1, it is defined as

$$
\operatorname{Pr}=\frac{\int_{\tau_{1}}^{\tau_{\mathrm{cut}}} C_{b, 1}(\tau, x=1) d \tau}{\tau_{\mathrm{cyc}}} .
$$

This reduced productivity can be be easily back transformed in the usual dimensional form by multiplying with the volumetric flow rate. The recovery yield is the ratio of between the amount of desired component in purified fraction and the amount injected at the column inlet. For component 1, it is expressed as

$$
\mathrm{Y}=\frac{\int_{\tau_{1}}^{\tau_{\text {cut }}} C_{b, 1}(\tau, x=1) d \tau}{\int_{\tau_{1}}^{\tau_{2}} C_{b, 1}(\tau, x=1) d \tau}
$$

\section{Numerical case studies}

This section presents some numerical test problems to analyze the effects of $\rho_{\text {core }}, P e$ and $B i$ on the elution profiles. Moreover, the effects of $\rho_{\text {core }}, C_{i, \text { inj }}$, and $B i$ are analyzed on productivity and yield. The representative parameters used for illustration in the test problems are given in Table 1. 


\subsection{Single-component elution}

Figure 2(a) shows a comparison of the concentration profiles for different core radius fractions including fully porous beads. On increasing $\rho_{\text {core }}$ from 0 (fully porous beads) to 0.8 (beads with thin shell), the elution profiles became sharpened. Thus, with an increase in

$\rho_{\text {core }}$, efficiency of the column improves. Simultaneously, the retention time of the column shortens, i.e. the capacity of the column reduces. The sharpening of the peaks are due to the reduced intraparticle diffusional mass transfer resistance. The shorter residence times are due to the loss of binding sites with increasing the $\rho_{\text {core }}$ value. Figure 2(b) compares the results for linear $(b=0)$ and nonlinear $(b=10)$ cases. The nonlinear effects caused further reduction in the retention times and more pronounced peak tailings. The effects of model parameters $P e$ and $B i$ on the elution curves are also shown in Figures 2(c) and 2(d) for two different values of $\rho_{\text {core }}$. It is apparent that if axial dispersion or film mass transfer resistance are important, the peaks become wider and the times corresponding to the peak maxima are slightly lower. The effect of $\rho_{\text {core }}$ is similar in all plots.

\subsection{Multi-component elution}

Figure 3 shows the effect of $\rho_{\text {core }}$ on the retention times and band broadening of the elution profiles for a two-component mixture with $b_{1} C_{b, 1}^{\mathrm{inj}}=b_{2} C_{b, 2}^{\mathrm{inj}}=1$. It can be observed again that for fully porous particles the overlap in the elution profiles is rather significant. Thus, the separation of the two peaks is not achieved in this case. However, for core-beads with $\rho_{\text {core }}=0.8$ elution peaks are sharper, retention times of both components are shorter and the resolution is much better. It can be observed that at $\rho_{\text {core }}=0.8$ separation of the two 
peaks is almost achieved due to decrease in band broadening. The aggregate dimensionless time required for totally eluting out the two peaks was reduced from 42 for fully porous beads (c.f. Figure 3) to 16 for cored beads having $\rho_{\text {core }}=0.8$, offering larger productivities in a repetitive batch regime.

The peak areas of both components for $\rho_{\text {core }}=0.8$ were numerically integrated and were found to be 0.998 and 0.995 , respectively. They matched well with the dimensionless sample pulse size of one injected for $\tau_{\text {inj }}=1.0$. Moreover, the numerical dispersion of the scheme was very small and elution profiles on course and refined grids were almost the same. These tests verify the accuracy of proposed numerical algorithm.

Figure 4 shows the effect of $\rho_{\text {core }}$ on retention times and band broadening of the elution profiles for a three-component mixture. It can be observed in Figure 4a that fully porous particles did not provide separation of the three peaks. It was also found that cored beads with $\rho_{\text {core }}=0.5$ did not help much in further improving the separation. However, as $\rho_{\text {core }}$ was increased to 0.8 , elution peaks became sharper (c.f. Figure $4 \mathrm{~b}$ ) and retention times of all three components were shorter. With this value of $\rho_{\text {core }}$, separation of the three peaks could be achieved. The aggregate dimensionless time required for totally eluting the three peaks was roughly 80 for fully porous beads (c.f. Figure 4), compared to 35 for cored beads having $\rho_{\text {core }}=0.8$.

The peak areas of three components for $\rho_{\text {core }}=0.8$ were numerically integrated and were found to be 1.001, 1.002 and 1.0, respectively. They matched well with the dimensionless sample pulse size of 1.0 for $\tau_{\mathrm{inj}}=1$. This verifies the accuracy of proposed numerical method. Our numerical results are also in good agreement with those obtained by Gu et 
al. (2011) for the same data.

\subsection{Assessment of the process performance}

Figure 5 shows the plot of $\tau_{\text {cyc }}, \tau_{\text {cut }}$, Pr, and $Y$ (c.f. (33)-(36)) over $\rho_{\text {core }}$. It can be observed that the cycle time is decreasing from 42 for fully porous beads to 12 for cored beads having $\rho_{\text {core }}=0.85$. Similarly, the cut times reduce from 9.7 to 5.6. The productivity increases till $\rho_{\text {core }}=0.7$ and decreases afterwards. On the other hand, the yield $Y$ increases continuously with increasing $\rho_{\text {core }}$. It should be noted that the characteristic maximum visible in Figure $5 \mathrm{c}$ is just valid for the specific parameters considered. Below we will illustrate the effect of selected parameters on the course of the performance criteria.

Figure 6 shows the effect of the injection concentration $C_{b, 1}^{\text {inj }}$ on the parameters $\tau_{\text {cyc }}, \tau_{\text {cut }}$, Pr, and $Y$ (c.f. Eqs. (33)-(36)) for four different values of $\rho_{\text {core }}$. Here, we have chosen $C_{b, 1}^{\mathrm{inj}}=C_{b, 2}^{\mathrm{inj}}$. On increasing $C_{b, 1}^{\mathrm{inj}}$ the productivity initially increases and achieves a maximum value. Afterwards, it decreases and finally attains a steady state level. Table 2 lists the maximum values of productivity along with other parameters at the given values of $\rho_{\text {core }}$ and $C_{b, 1}^{\text {inj. }}$. It can be seen in Table 2 and Figure 6 that maximum values of productivity were achieved in the range $1.0 \mathrm{~g} / \mathrm{l} \leq c_{\mathrm{inj}} \leq 3.0 \mathrm{~g} / \mathrm{l}$ for $0 \leq \rho_{\text {core }} \leq 0.8$. In this range, for $\rho_{\text {core }}=0.7$ and $C_{b, 1}^{\text {inj }}=3 \mathrm{~g} / l$ the maximum productivity is found. It should be noted that an increase in the feed concentration is equivalent to the increase of $b_{i}$ as shown in the isotherm expression given by Eq. (7).

To illustrate the effects of the thickness of the core and the injection concentrations, Figure 7 displays the elution curves at maximum levels of productivity achieved at particular 
values of $\rho_{\text {core }}$ and $C_{b, 1}^{\text {inj }}=C_{b, 2}^{\text {inj }}$ (c.f. Table 2 and Figure 6$)$.

We further evaluated the effects of two kinetic parameters, namely a) the external mass transfer resistance, expressed via $B i$, and b) the intraparticle diffusion resistance, expressed via $\eta$.

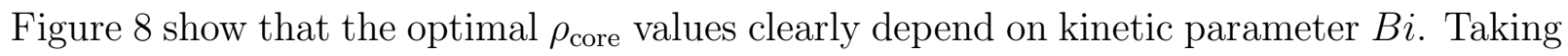
the results of Figures $5(\mathrm{c}),(\mathrm{d})$ generated for $B i=50$ as a reference, two other Biot numbers were considered ( $B i=5$ and $B i=150$ ). A decrease and an increase of the mass transfer rate around the particles changed in the same way the ratio between external and internal mass transfer resistances. Figure $8(\mathrm{a})$ shows that the optimal $\rho_{\text {core }}$ values, which lead to the highest productivity for collecting the first eluting component, move to larger values on decreasing the $B i$, i.e. to thinner shell layers. Simultaneously, the recovery drops with a decrease in mass transfer rate. The differences in productivity and yield for Biot numbers of 50 or 150 are small. This indicates that for $B i=50$ the effect of the transport through the laminar boundary layer is already almost negligible. Band shapes are under such conditions essentially controlled be internal mass transfer resistances and axial dispersion. Finally, results in Figure 9 show the dependence of optimal $\rho_{\text {core }}$ values on parameter $\eta$. Once again, taking the results of Figures $5(\mathrm{c}),(\mathrm{d})$ generated for $\eta=2.0$ as a reference, two other $\eta$ numbers were considered (i.e. $\eta=0.5,2.5)$. Figure 9 (a) shows that the optimal $\rho_{\text {core }}$ values, which lead to the highest productivity for collecting the first eluting component, move to lower values on increasing the $\eta$. For $\eta=2.5$ the optimum $\rho_{\text {core }}$ is roughly 0.5. Thus, faster transfer rates in the shell allow to thicken this layer. Simultaneously, the recovery drops monotonously with a decrease in $\eta$ value. It should be emphasized that 
these results and the results shown in Figure 8 are just valid for the given feed composition. Changes in the optima would occur for other injections concentrations as illustrated in Figure 6.

With the model applied and the accurate solution method investigated the impact of all other relevant parameters could be studied in detail. The selected case studies of this manuscript clearly indicate that a full optimization of core-shell particles for applications in nonlinear preparative chromatography is a challenging task. For application in preparative chromatography a rational optimization needs to carefully consider, besides the geometric, kinetic and thermodynamic parameters, also the operating parameters, in particular the injection concentrations.

\subsection{Conclusion}

A semi-discrete high resolution finite volume scheme was proposed to solve the nonlinear general rate model for fixed-beds packed with core shell adsorbents. It quantifies the effects of intraparticle diffusion resistance, film mass transfer resistance and the axial dispersion influence on the shape of elution curves. The results showed that an increase in core radius fraction leads to shorter residence times and sharper peaks. Thus, if a column is packed with core shell adsorbents, the column separation efficiency will increase due to the shortened diffusion path in the adsorbents. In contrast the loading capacity decreases. Suitable performance criteria considering the cycle times were evaluated exploiting numerical simulations for finding the optimum values of core radius fraction and injected concentration. Selected parametric studies revealed that the optimal shell layer thickness which maxi- 
mizes the productivity of preparative chromatography is, among others, a function of the injection concentration, the Biot number and the intraparticle diffusion resistance. The presented model and the numerical solutions are seen to be useful to understand and optimize the process and to develop proper core shell particles.

Acknowledgement: The first author gratefully acknowledge the Higher Education Commission (HEC) of Pakistan for financial support.

\section{References}

Ali, I., Al-Othman, Z.A., Al-Záabi, M., 2012. Superficially porous particles columns for super fast HPLC separations, Biomedical Chromatography 26, 1001-1008.

Cabootera, D., Fanigliulob, A., Bellazzi, G., Allieri, B., Rottigni, A., Desmeta, G., 2010. Relationship between the particle size distribution of commercial fully porous and superficially porous high-performance liquid chromatography column packings and their chromatographic performance. Journal of Chromatography A 1217, 7074-7081.

Carta, G., 1988. Exact analytical solution of a mathematical model for chromatographic operations. Chemical Engineering Science 43, 2877-2883.

Coutinho, F.M.B., Carvalho,D.L., La Torre Aponte,M.L.,Barbosa,C.C.R., 2001. Pellicular ion exchange resins based on divinylbenzene and 2-vinylpyridine. Polymer 42, 43-48.

Danckwerts, P.V., 1953. Continuous flow systems. Chemical Engineering Science 2, 1-9.

Fanigliulo, A., Cabooter, D., Bellazzi, G., Tramarin, D., Allieri, B., Rottigni, A., Desmet, 
G., 2010. Comparison of performance of high performance liquid chromatography columns packed with superficially and fully porous $2.5 \mathrm{~mm}$ particles using kinetic plots. Journal of Separation Science 33, 3655-3665.

Fekete, S., Ganzler, K., Fekete, J., 2010. Facts and myths about columns packed with sub-3 $\mu m$ and sub-2 $\mu m$ particles. Journal of Pharmaceutical and Biomedical Analysis $51,56-64$.

Greibrokk, T., 2004. The contributions of Csaba Horvth to liquid chromatography. Journal of Separation Science 27, 1249-1254.

Gritti, F., Leonardis, I., Shock, D., Stevenson, P., Shalliker, A., Guiochon, G., 2010. Performance of columns packed with the new shell particles, Kinetex-C18. Journal of Chromatography A 1217, 1589-603.

Gritti, F., Guiochon, G., 2010. Comparison of heat friction effects in narrow-bore columns packed with coreshell and totally porous particles. Chemical Engineering Science 65, 6310-6319.

González-Ruiz, V., Olives, A.I., Martín, M.A., 2015. Core-shell particles lead the way to renewing high-performance liquid chromatography, Trends in Analytical Chemistry 64, $17-28$.

Gu, T., Liu, M., Cheng, K.-S.C., Ramaswamy, S., Wang, C., 2011. A general rate model approach for the optimization of the core radius fraction for multicomponent isocratic 
elution in preparative nonlinear liquid chromatography using cored beads. Chemical Engineering Science 66, 3531-3539.

Gu, T., Tsao, G.T., Tsai, G.-J., Ladisch, M.R., 1990. Displacement effect in multicomponent chromatography. Chemical AIChE 36, 1156-1162.

Guiochon, G., 2002. Preparative liquid chromatography. Journal of Chromatography A, $965,129-161$.

Guiochon, G., Felinger, A., Shirazi, D.G., Katti, A.M., 2006. Fundamentals of preparative and nonlinear chromatography, 2nd ed. ELsevier Academic press, New York.

Guiochon, G., Gritti, F., 2011. Shell particles, trials, tribulations and triumphs. Journal of Chromatography A 1218, 1915-38.

Guiochon, G., Lin, B., 2003. Modeling for preparative chromatography, Academic Press.

Hayesa, R., Ahmeda, A., Edge, T., Zhanga, H., 2014. Coreshell particles: Preparation, fundamentals and applications in high performance liquid chromatography. Journal of Chromatography A 1357, 36-52.

Horvath, C.G., Preiss, B.A., Lipsky, S.R., 1967. Fast liquid chromatography: an investigation of operating parameters and the separation of nucleotides on pellicular ion exchangers. Analytical Chemistry 39, 1422-1428.

Horváth, K, Felinger, A., 2015. Influence of particle size and shell thickness of core-shell 
packing materials on optimum experimental conditions in preparative chromatography. Journal of Chromatogrpahy A 1407, 100-105.

Javeed, S., Qamar, S., Seidel-Morgenstern, A., Warnecke, G., 2011. Efficient and accurate numerical simulation of nonlinear chromatographic processes. Computers \& Chemical Engineering 35, 2294-2305.

Kaczmarski, K., Guiochon, G., 2007. Modeling of the mass-transfer kinetics in chromatographic columns packed with shell and pellicular particles. Analytical Chemistry 79, 4648-4656.

Kirkland, J.J., Truszkowski, F.A., Dilks, C.H., Engel, G.S., 2000. Superficially porous silica microspheres for fas thigh-performance liquid chromatography of macromolecules. Journal of Chromatography A 890, 3-13.

Kirkland, J.J., Langlois, T.J., DeStefano, J.J., 2007. Fused core particles for HPLC columns. American Laboratory 39, 18-21.

Kiss, I., Bacskay, I., Kilár, F., Felinger, A., 2010. Comparison of the mass transfer in totally porous and superficially porous stationary phases in liquid chromatography. Analytical and Bioanalytical Chemistry 397, 1307-1314.

Lambert, N., Kiss, I., Felinger, A., 2014. Significance and estimation of chromatographic parameters. Journal of Chromatography A 1366, 84-91.

Lee, W.-C., 1997. Protein separation using non-porous sorbents. Journal of Chromatography B 699, 29-45. 
Li, P., Xiu, G., Rodrigues, A.E., 2003. Analytical breakthrough curves for inert core adsorbent with sorption kinetics, AIChE J., 49, 2974-2979.

Li, P., Xiu, G., Rodrigues, A.E., 2003. Modeling separation of proteins by inert core adsorbent in a batch adsorber. Chem. Eng. Sci., 58, 3361-3371.

Li, P., Xiu, G., Rodrigues, A.E., 2004. Modeling breakthrough and elution curves in fixedbed of inert core adsorbents: analytical and approximate solutions. Chem. Eng. Sci., 59, 3091-3103.

Li, P., Xiu, G., Rodrigues, A.E., 2005. Experimental and modeling study of protein adsorption in expanded bed adsorption process. AIChE J., 51, 2965-2977.

Li, P., Xiu, G., Mata, V., Grande, C., Rodrigues, A.E., 2006. Expanded Bed adsorption/desorption of proteins with streamline direct CST I adsorbents, Biotechnol. Bioeng., 94, 1155-1163.

Li, P., Yu, J., Xiu, G., Rodrigues, A.E., 2010. A strategy for tailored design of efficient and low-pressure drop packed column chromatography. AIChE Journal 56, 3091-3098.

Lieres, E.V., Andersson, J., (2010). A fast and accurate solver for the general rate model of column liquid chromatography. Computers \& chemical engineering 34, 1180-1191.

Luo, J., Zhou, W., Su, Z., Ma, G., Gu, T., (2013). Comparison of fully-porous beads and cored beads in size exclusion chromatography for protein purification. Chemical Engineering Science 102, 99-105. 
Manchon, N., Arrigo, M.D., Garcia-Lafuente, A., Guillamon, E., Villares, A., Ramos, A., Martinez, J.A., Rostagno, M.A., 2010. Fast analysis of isoflavones by high-performance liquid chromatography using a column packed with fused-core particles. Talanta 82, 1986-1994.

Miyabe, K., 2008. Evaluation of chromatographic performance of various packing materials having different structural characteristics as stationary phase for fast high performance liquid chromatography by new moment equations. Journal of Chromatography A 1183, 49-64.

Ning, J., Kong, F.Z., Li, D.H., Du, Y.Z., 1998. Preparation of monodisperse agglomerated pellicular anion-exchange resins compatible with high-performance liquid chromatography solvents for ion chromatography. Journal of Chromatography A 793, 193-197.

Pesek, J.J., Frost, J.H., 1973. Adsorption as a mechanism for separation of nonionic solutes by pellicular ion exchange chromatography. Analytical Chemistry 45, 1762-1765.

Pietrogrande, M.C., Dondi, F., Ciogli, A., Gasparrini, F., Piccin, A., Serafini, M., 2010. Characterization of new types of stationary phases for fast and ultra-fast liquid chromatography by signal processing based on AutoCovariance function: a case study of application to Passiflora incarnata L. extract separations. Journal of Chromatography A $1217,4355-4364$.

Qamar, S., Abbasi, J.N., Mehwish, A., 2015. Linear general rate model of chromatography 
for core-shell particles: Analytical solutions and moment analysis. Chemical Engineering Science, 137, 352-363.

Rissler, R., 2000. Separation of polyesters by gradient reversed-phase high- performance liquid chromatography on a $1.5 \mu m$ non-porous column. Journal of Chromatography A 871, 243-258.

Ruthven, D.M., 1984. Principles of adsorption and adsorption processes. WileyInterscience, New York.

Salisbury, J.J., 2008. Fused-core particles: a practical alternative to sub-2 micron particles, Journal of Chromatographic Science 46, 883-886.

Spudeit, D.A., Dolzan, M.D., Breitbach, Z.S., Barber, W.E., Micke, G.A., Armstrong D.W., 2014. Superficially porous particles vs. fully porous particles for bonded high performance liquid chromatographic chiral stationary phases: isopropyl cyclofructan 6 . Journal of Chromatography A 1363, 89-95.

Wang, X.L., Barber, W.E., Carr, P.W., 2006. A practical approach to maximizing peak capacity by using long columns packed with pellicular stationary phases for proteomic research. Journal of Chromatography A 1107, 139-151.

Xiang, Y.Q., Yan, B.W., McNeff, C.V., Carr, P.W., Lee, M.L., 2003. Synthesis of micron diameter polybutadiene-encapsulated non-porouszirconia particles for ultrahigh pressure liquid chromatography. Journal of Chromatography A 1002,71-78. 
Zhou, X., Sun, Y., Liu, Z., 2007. Superporous pellicular agaroseglass composite particle for protein adsorption. Biochemical Engineering Journal 34, 99-106. 
Table 1: Standard parameters of the problems.

\begin{tabular}{|c|c|c|c|c|c|c|c|c|c|}
\hline Figure Nr. & Component Nr. & $P e_{i}$ & $B_{i}$ & $\eta_{i}$ & $\epsilon_{b}$ & $\epsilon_{p}$ & $a_{i}$ & $b_{i}$ & $C_{b, i}^{\mathrm{inj}}$ \\
\hline 2 & 1 & 1500 & 50 & 2.0 & 0.4 & 0.5 & 10 & 1.0 & 1.0 \\
\hline \multirow[t]{2}{*}{3} & 1 & & & & & & 10 & 1.0 & 1.0 \\
\hline & 2 & 1500 & 50 & 2.0 & 0.4 & 0.5 & 25 & 1.0 & 1.0 \\
\hline \multirow[t]{3}{*}{4} & 1 & & & & & & 10 & 0.5 & 0.1 \\
\hline & 2 & & & & & & 30 & 1.5 & 0.4 \\
\hline & 3 & 1500 & 50 & 2.0 & 0.4 & 0.5 & 70 & 3.5 & 0.4 \\
\hline \multirow[t]{2}{*}{$5,6 \& 7$} & 1 & & & & & & 10 & 1.0 & 1.0 \\
\hline & 2 & 1500 & 50 & 2.0 & 0.4 & 0.5 & 25 & 1.0 & 1.0 \\
\hline \multirow[t]{2}{*}{8} & 1 & & & & & & 10 & 1.0 & 1.0 \\
\hline & 2 & 1500 & $(5,50,150)$ & 2.0 & 0.4 & 0.5 & 25 & 1.0 & 1.0 \\
\hline \multirow[t]{2}{*}{9} & 1 & & & & & & 10 & 1.0 & 1.0 \\
\hline & 2 & 1500 & 50 & $(0.5,2,2.5)$ & 0.4 & 0.5 & 25 & 1.0 & 1.0 \\
\hline
\end{tabular}


Table 2: Optimum values of assessment parameters.

\begin{tabular}{cccccc}
\hline$\rho_{\text {core }}$ & $C_{b, 1}^{\text {inj }}$ & $\operatorname{Pr}$ & $Y$ & $\tau_{\text {cyc }}$ & $\tau_{\text {cut }}$ \\
\hline \hline 0.0 & 3.0 & 0.044 & 0.60 & 39.96 & 5.18 \\
0.5 & 3.0 & 0.062 & 0.66 & 31.31 & 4.45 \\
0.7 & 3.0 & 0.088 & 0.76 & 21.26 & 4.01 \\
0.8 & 1.0 & 0.048 & 0.99 & 14.71 & 5.90 \\
\hline
\end{tabular}

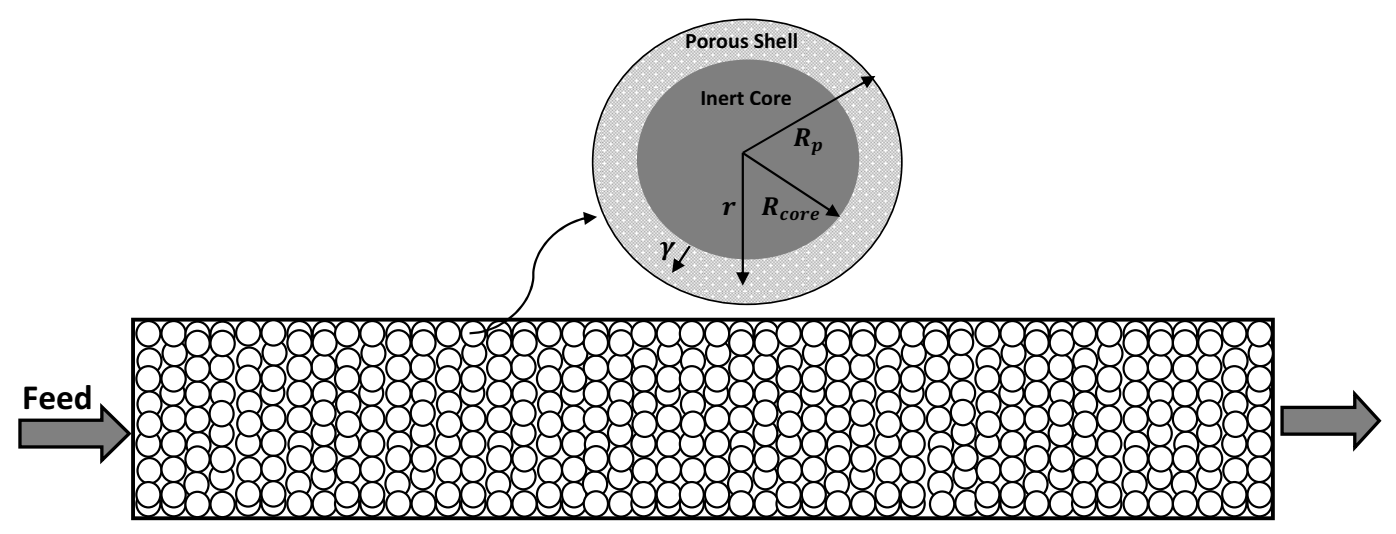

Figure 1: Schematic diagrams of fixed-bed adsorber and inert core adsorbent. 
(a)

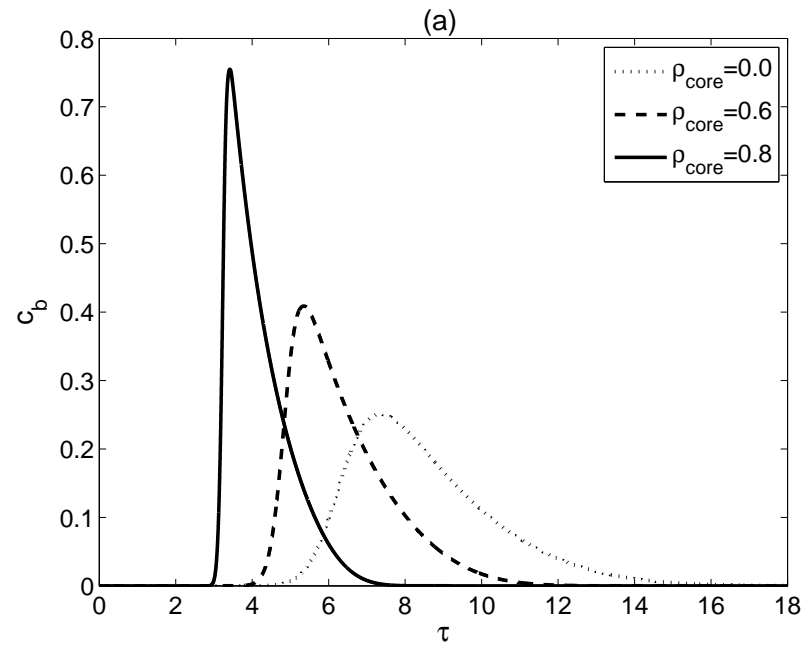

(c)

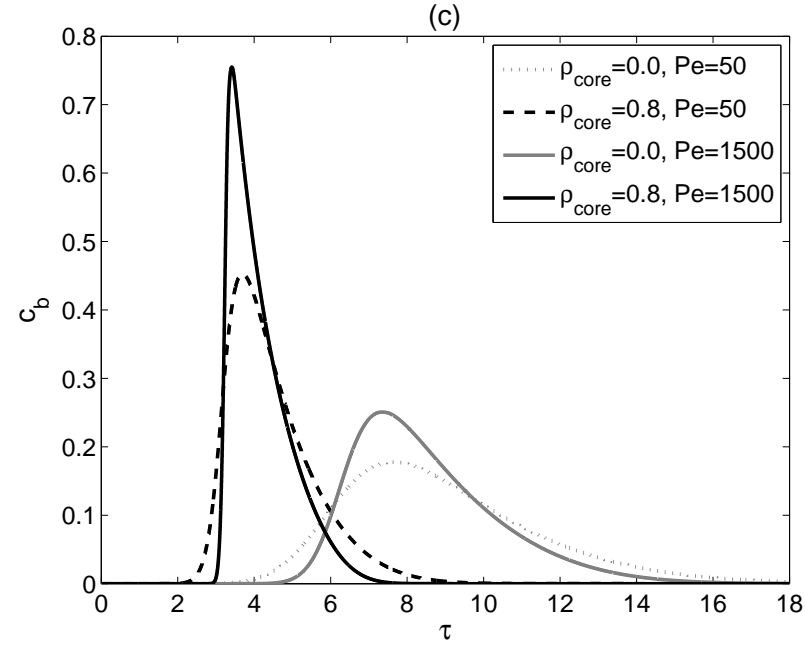

(b)

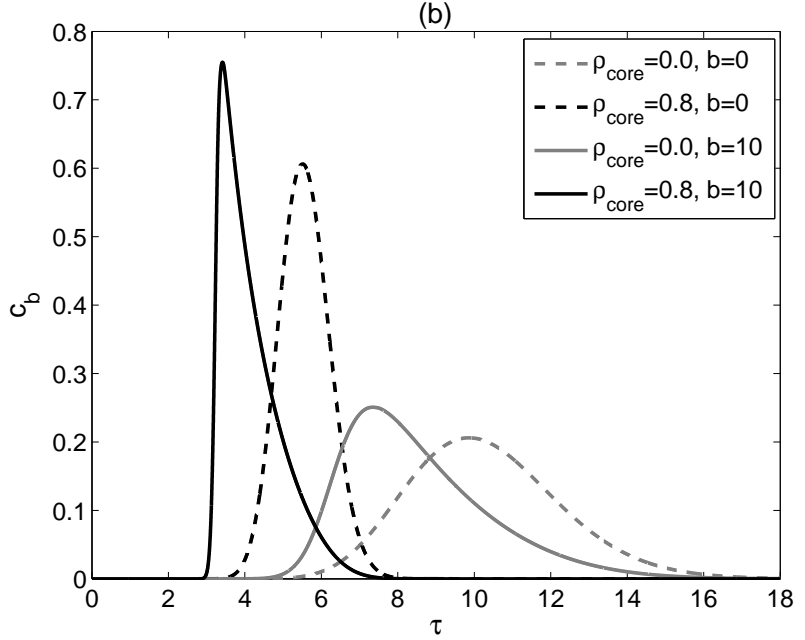

(d)

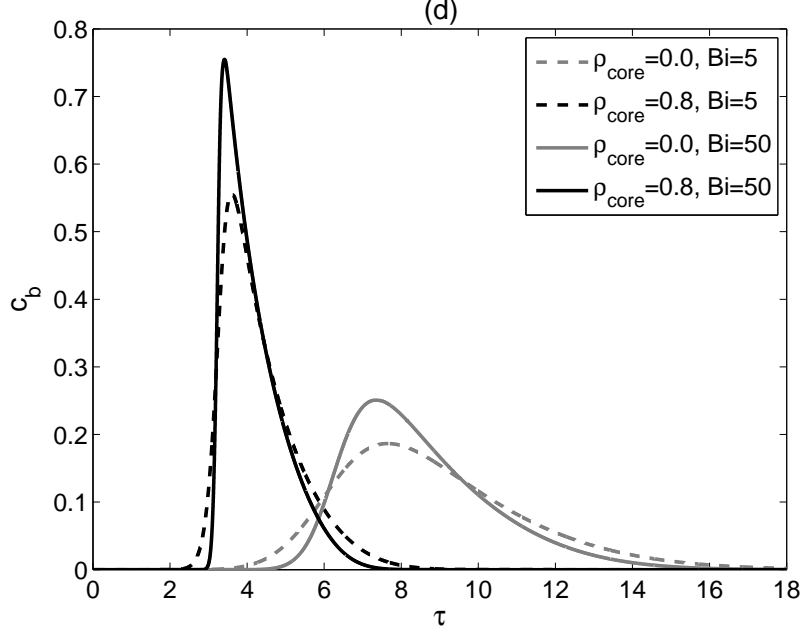

Figure 2: Single solute: Study of the effects of (a) $\rho_{\text {core }}$, (b) extent of nonlinearity $b$, (c) $P e$, and (d) $B i$ on elusion curves. Here, $\tau_{\mathrm{inj}}=1$ and values of the standard parameters are given in Table 1 . 
(a)

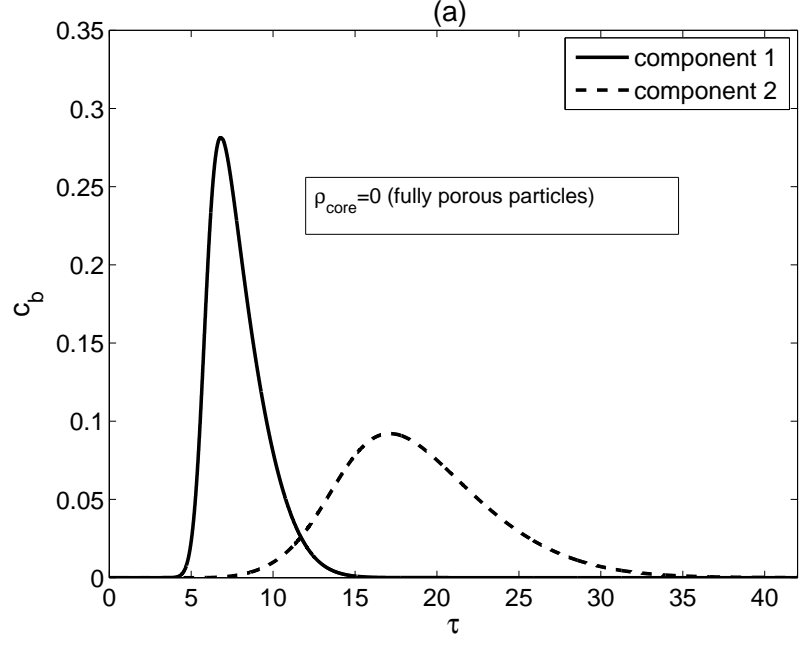

(b)

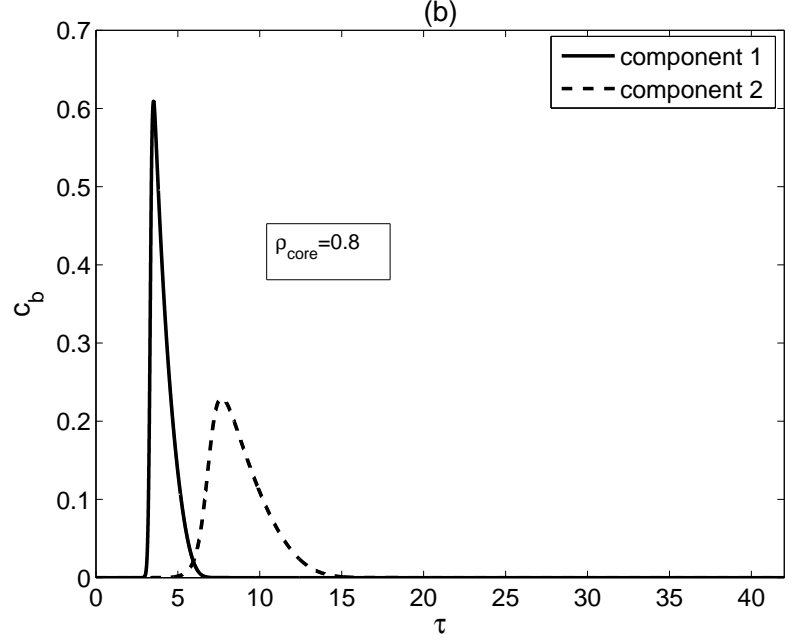

Figure 3: Two-component mixture: Elution profiles at the column outlet for (a) fully porous particles and (b) cored beads for $b_{1} C_{b, 1}^{\mathrm{inj}}=1=b_{2} C_{b, 2}^{\mathrm{inj}}$. The values of standard parameters are given in Table 1 .

(a)

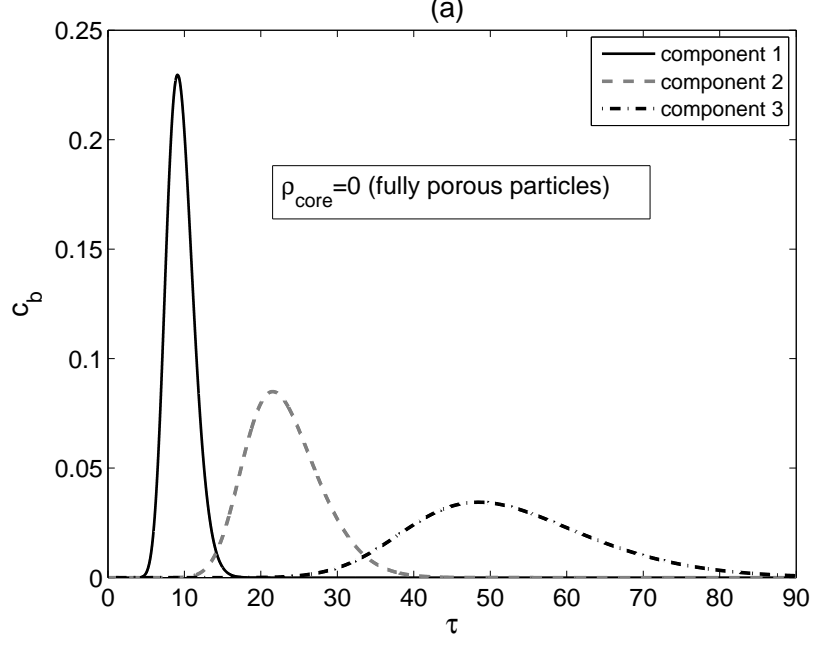

(b)

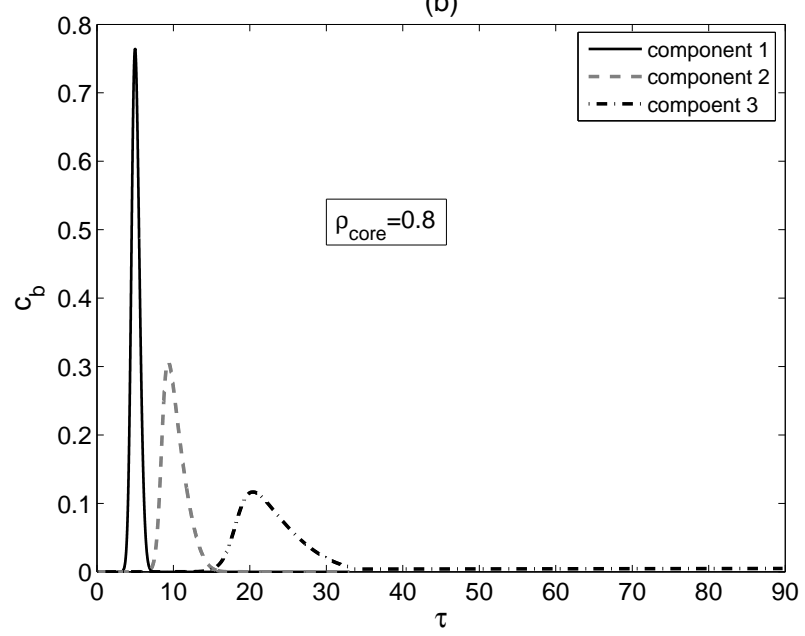

Figure 4: Three-component mixture: Elution profiles at the column outlet for (a) fully porous particles and (b) cored beads for $\tau_{\mathrm{inj}}=1$. The values of standard parameters are given in Table 1 . 
(a)

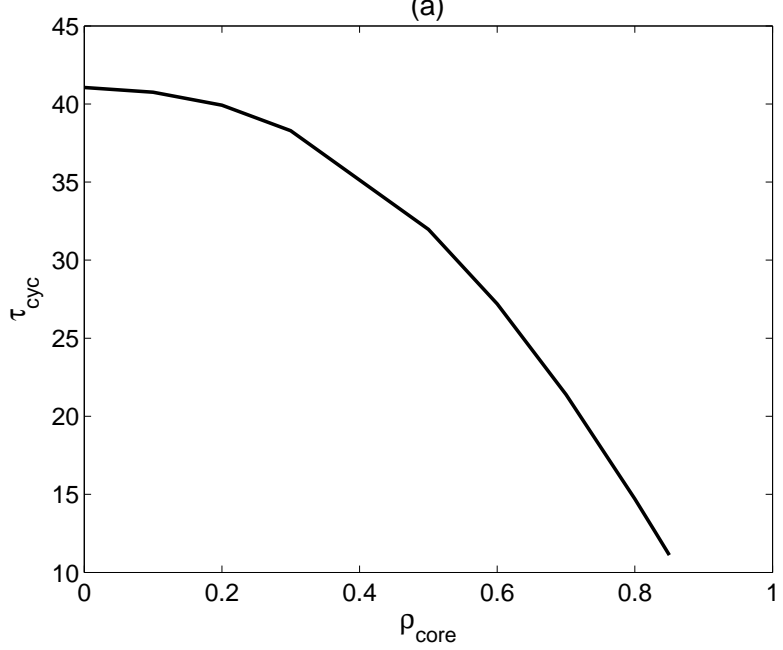

(c)

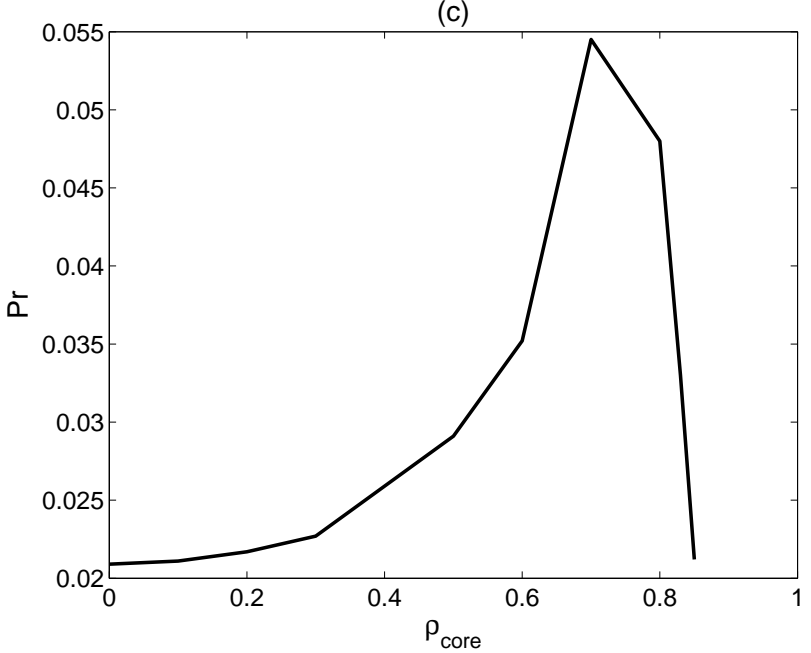

(b)

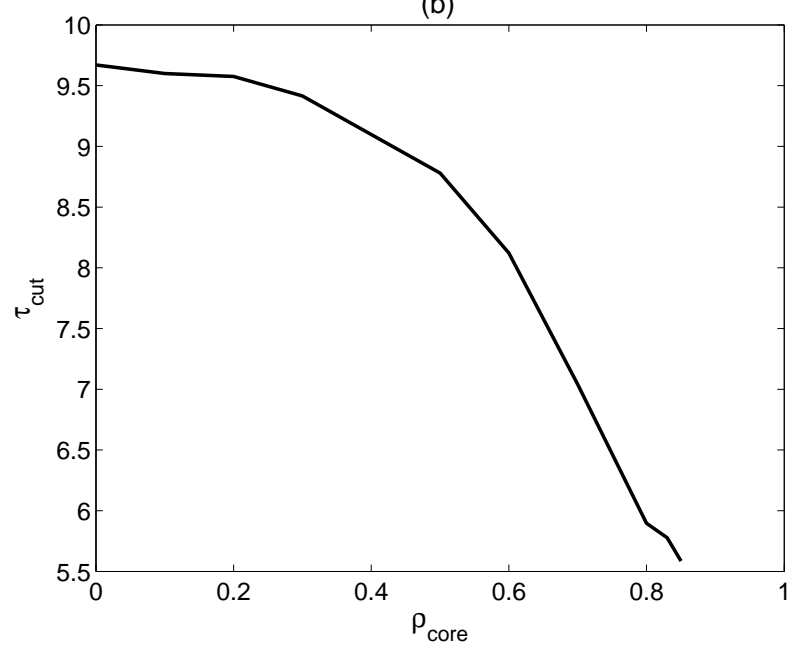

(d)

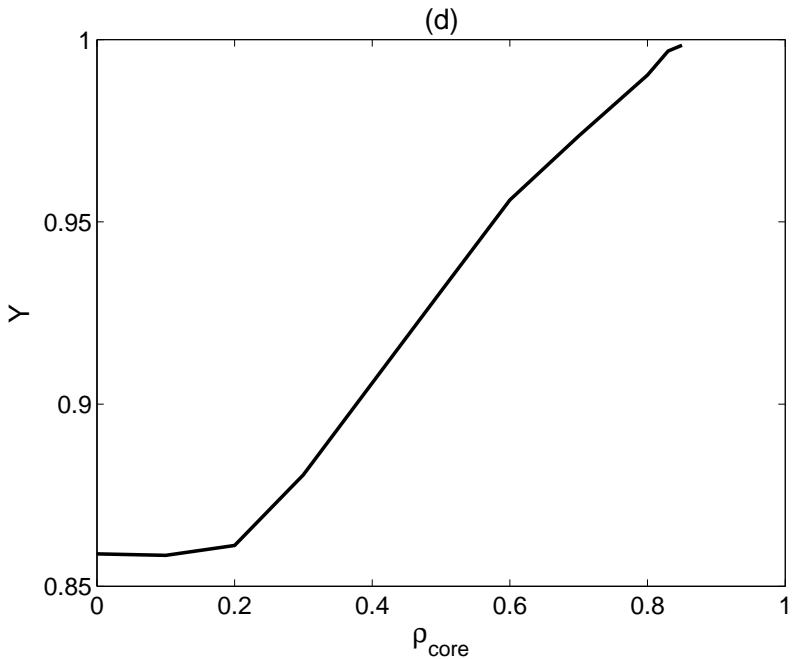

Figure 5: Two-component mixture: Plots of (a) $\tau_{\text {cyc }}$, (b) $\tau_{\text {cut }}$, (c) Pr, and (d) $Y$ as functions of $\rho_{\text {core }}$ for fixed values of $b_{1} C_{b, 1}^{\mathrm{inj}}=1=b_{2} C_{b, 2}^{\mathrm{inj}}$ and $\tau_{\mathrm{inj}}=1.0$. The values of standard parameters are given in Table 1 . 

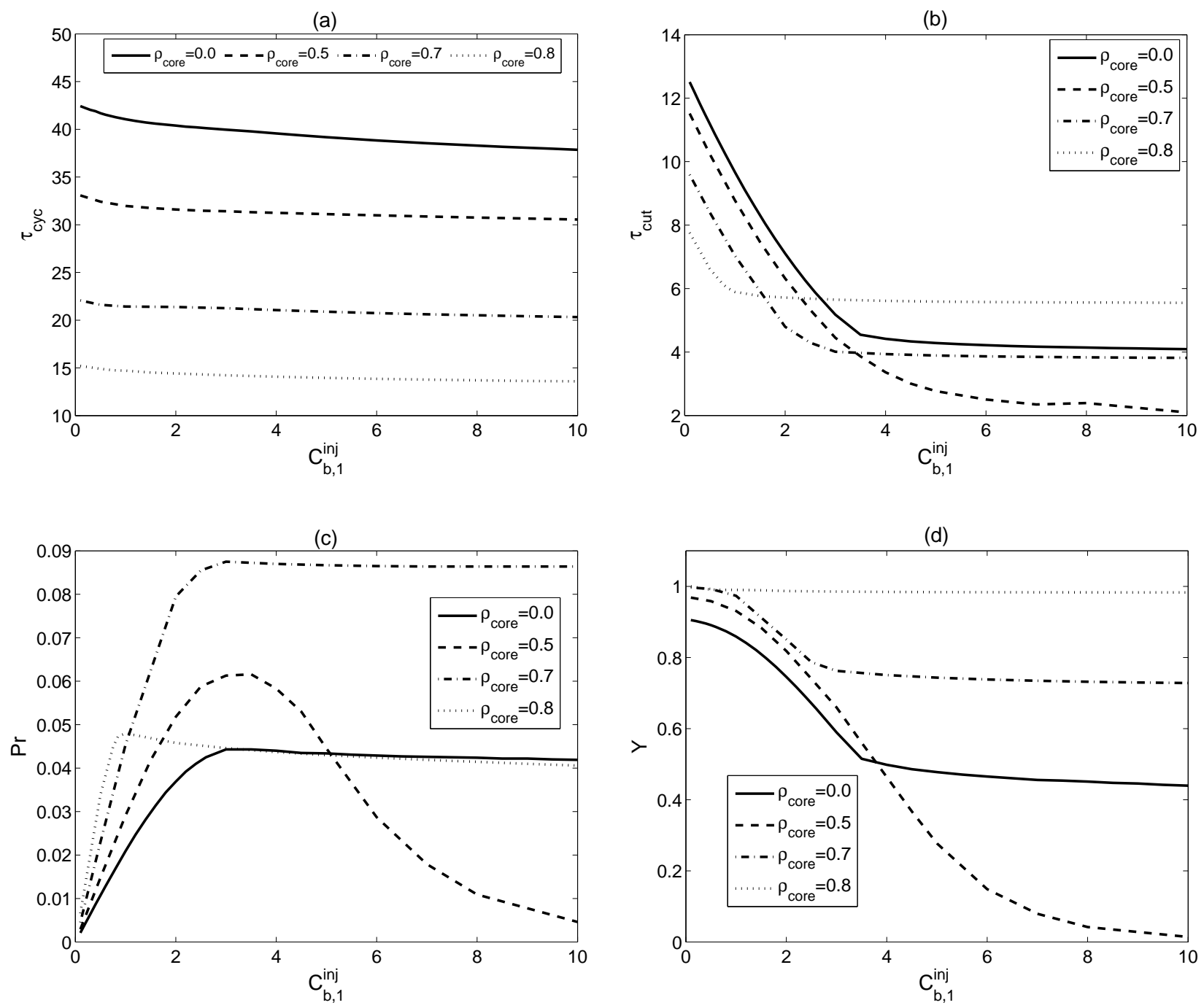

Figure 6: Two-component mixture: Plots of (a) $\tau_{\text {cyc }}$, (b) $\tau_{\text {cut }}$, (c) Pr, and (d) $Y$ as functions of $C_{b, 1}^{\text {inj }}$ for different values of $\rho_{\text {core }}$. Here, $\tau_{\text {inj }}=1.0, C_{b, 1}^{\text {inj }}=C_{b, 2}^{\text {inj }}$ and $b_{1}=1=b_{2}$. The values of standard parameters are given in Table 1. 
(a)

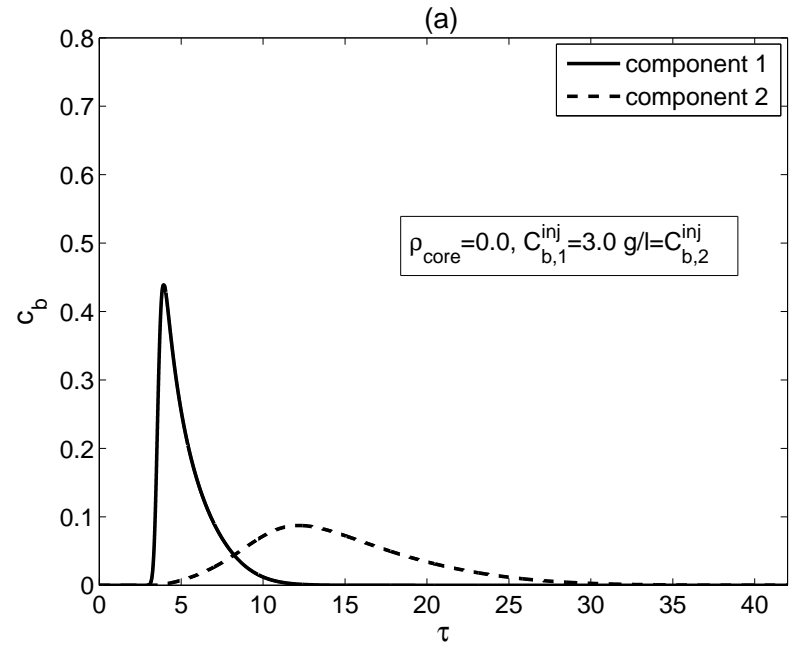

(c)

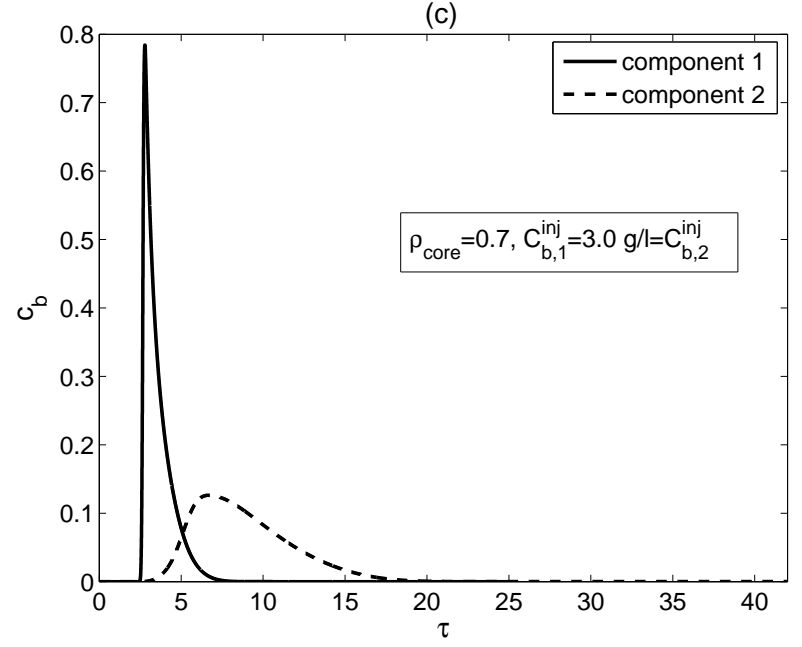

(b)

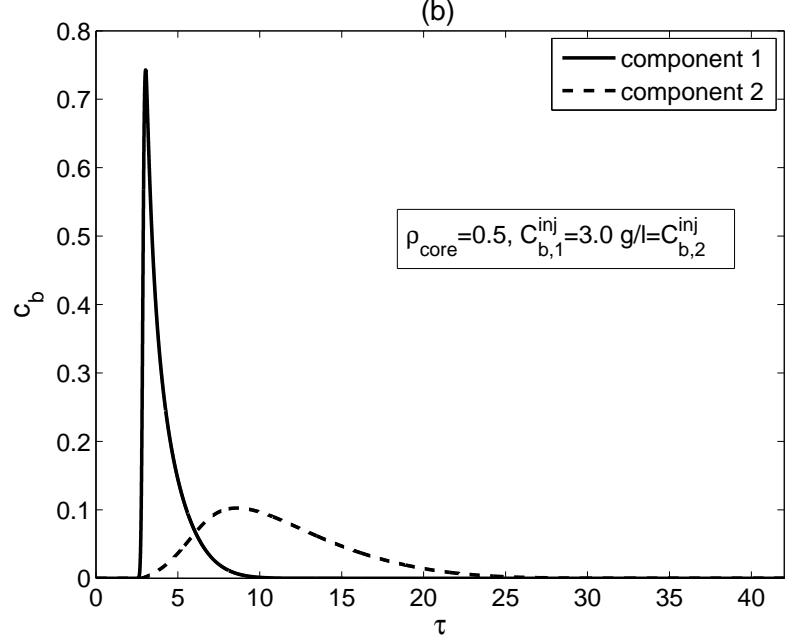

(d)

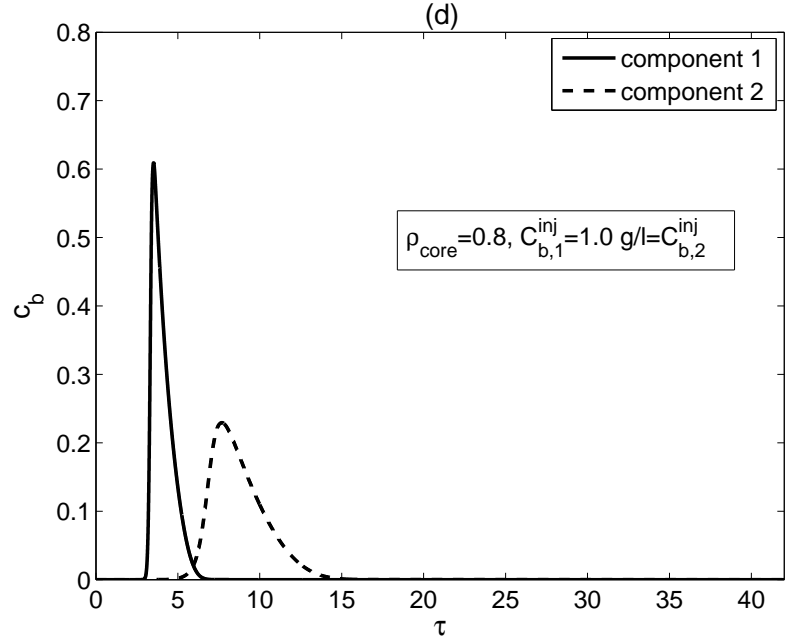

Figure 7: Two-component mixture: Plots plots (a), (b), (c) and (d) show comparisons of elution profiles at maximum productivity levels for particular $\rho_{\text {core }}$ and $C_{b, i}^{\text {inj }}$ (c.f. Figure $\left.6(\mathrm{~b})\right)$. Here, $\tau_{\text {inj }}=1.0$ and $b_{1}=1=b_{2}$. The values of standard parameters are given in Table 1 . 
(a)

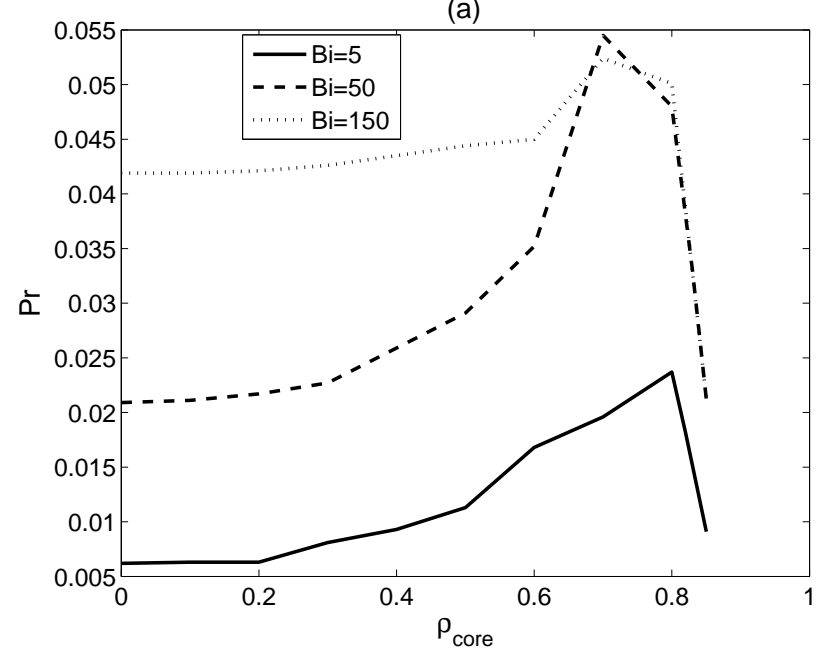

(b)

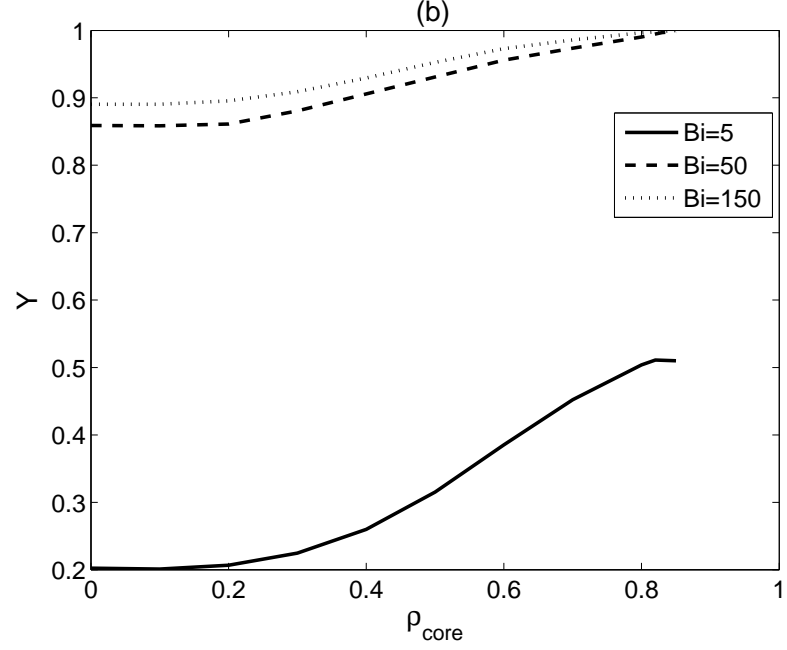

Figure 8: Two-component mixture: Plots of (a) Pr and (b) $Y$ as functions of $\rho_{\text {core }}$ for three different values of $B i\left(5,50\right.$ (Figure 5), 150) and fixed values of $b_{1} C_{b, 1}^{\mathrm{inj}}=1=b_{2} C_{b, 2}^{\mathrm{inj}}$ and $\tau_{\mathrm{inj}}=1.0$. The values of standard parameters are given in Table 1.
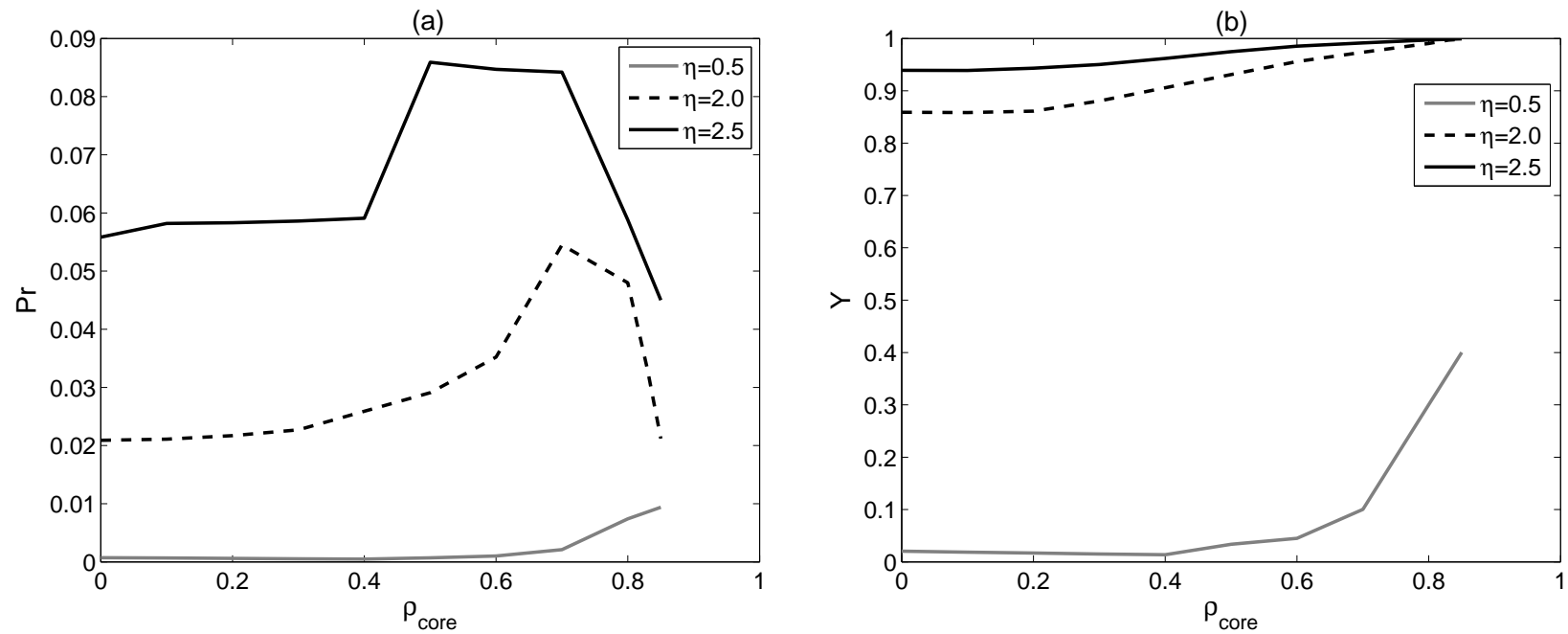

Figure 9: Two-component mixture: Plots of (a) $\operatorname{Pr}$ and (b) $Y$ as functions of $\rho_{\text {core }}$ for three different values of $\eta\left(0.5,2\right.$ (Figure 5), 2.5) and fixed values of $b_{1} C_{b, 1}^{\mathrm{inj}}=1=b_{2} C_{b, 2}^{\mathrm{inj}}$ and $\tau_{\mathrm{inj}}=1.0$. The values of standard parameters are given in Table 1. 